\title{
Exchange instabilities in electron systems: Bloch versus Stoner Ferromagnetism
}

\author{
Ying Zhang and S. Das Sarma \\ Condensed Matter Theory Center, Department of Physics, \\ University of Maryland, College Park, MD 20742-4111
}

(Dated: August 3, 2021)

\begin{abstract}
We show that $2 \mathrm{D}$ and $3 \mathrm{D}$ electron systems with the long-range Coulomb electron-electron interaction could develop ferromagnetic instabilities due to strong exchange effects at low densities. The critical densities in both 2D and 3D systems at which the magnetic instability, which could either be of Stoner type (second-order) or of Bloch type (first-order), are higher than the dispersion instability critical density where effective mass at the Fermi surface diverges. We discuss the theoretical as well as experimental implications of the ferromagnetic instability at low electron densities, particularly in low-disorder semiconductor-based two-dimensional systems.

PACS numbers: 71.10.-w; 71.10.Ca; 73.20.Mf; 73.40.-c
\end{abstract}

\section{INTRODUCTION}

With the development of high mobility semiconductor based 2D electron systems such as Si MOSFETs and GaAs HIGFETs, where extremely low carrier densities and very high quality can be achieved, a number of recent experiments has been carried out to measure fundamental physical quantities such as magnetic sus-

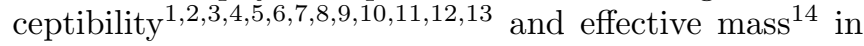
low density 2D electron systems at low temperatures $(\lesssim 100 \mathrm{mK})$. These experiments have triggered many theoretical as well as experimental studies on 2D and 3D electron systems. One important purpose of these studies is to obtain the ground state phase diagram for such systems. It is well known that the zero temperature 2D and $3 \mathrm{D}$ electron systems can be characterized by a single dimensionless interaction parameter $r_{s}=E_{e-e} / E_{K}$ (where $E_{e-e}$ denotes the interaction energy and $E_{K}$ the kinetic energy), which depends only on the density $(n)$ of the system with $r_{s} \propto n^{-1 / 2}\left(n^{-1 / 3}\right)$ in 2D (3D) systems. For small $r_{s}$, electron systems can be well described by Landau's Fermi liquid theory. (Actually, there is an exception even to this as at exceptionally low temperatures an interacting Fermi liquid undergoes a Khon-Luttinger superconducting transition, which we ignore for our purpose since it is of no physical relevance.) In the limit of large $r_{s}$, the system tends to reduce its interaction energy $E_{e-e}$ at the cost of higher kinetic energy by forming into an electron crystal (the so called Wigner crysta ${ }^{15}$ ), which has been established by Monte Carlo studies in both $2 \mathrm{D}^{16}$ (where the crystallization transition happens at $r_{s} \sim 32-42$ ) and $3 \mathrm{D}$ systems 17 (where the transition happens at $\left.r_{s} \sim 55-75\right)$. How the system behaves in between the above mentioned two limits of $r_{s}$ is not yet clear. It has been widely accepted, and also suggested by Monte Carlo studies, that there may exist a ferromagnetic phase (of either fully or partially polarized spins) in the intermediate $r_{s}$ region. Many other theoretical studies for such high $r_{s}$ value region typically start with a more or less arbitrary assumption of a particular ground state symmetry of the system. Among the various model of exotic interaction-driven electronic ground states, charge or spin density wave states, various superconducting states, glassy or clustered ground states have been much discussed in the literature.

From a theoretical perspective, it is important to study the evolution of the Fermi liquid state starting from the weakly interacting (small $r_{s}$ ) regime. As $r_{s}$ increases, all the single particle properties of the system are increasingly renormalized by interaction effects. The question is whether the Coulomb interaction renormalization brings about certain instabilities eventually at some large $r_{s}$, and hence changes the ground state symmetry. The existence of degrees of freedom related to spin and momentum makes it natural to consider the possibility of a magnetic instability or an instability that is related to the dispersion of a quasiparticle, which we call "dispersion instability" for short. In spite of a great deal of past theoretical work investigating the magnetic instabil-

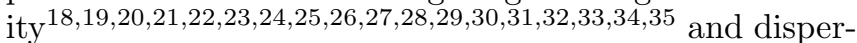
sion instability possibilities ${ }^{36.37}$ in both $2 \mathrm{D}$ and $3 \mathrm{D}$ electron systems using a large variety of different theoretical techniques, a unified theoretical treatment of the ferromagnetic instability, starting from the weak-coupling Fermi liquid ground state with interaction effects introduced systematically, is still lacking. This absence of a unified picture has left many unanswered important questions. First, what is the order of any possible ferromagnetic instability? It could be a second-order transition (the so-called Stoner instability) caused by the continuous divergence of the susceptibility as the critical density or $r_{s}$-value is approached from the weak-coupling side. Or it could be a first-order transition (the so-called Bloch ferromagnetism) which could happen abruptly at a specific $r_{s}$-value. Both have been predicted and studied in the literature, but their inter-relationship has not been clarified. Second, what is the relationship between magnetic instability and dispersion instability? Whether the possible divergence of the interacting spin susceptibility $\chi^{*}$, which can be written as $\chi^{*}=g^{*} m^{*}$, where $m^{*}\left(g^{*}\right)$ are the effective mass ( $g$-factor) of the system, is caused by the divergence of $m^{*}$ (dispersion instability) or $g^{*}$, or both? In order to answer these questions, we provide in this paper a comprehensive picture of the Bloch ferro- 
magnetic instability, Stoner ferromagnetic instability and the dispersion instability of the 2D and 3D electron system using the method of Random Phase Approximation $(\mathrm{RPA})$ 38.

The structure of the paper is the following: in Section [I] we discuss briefly the background for Bloch and Stoner ferromagnetism; in 【II we describe our theoretical formalism; in IV we present and discuss our theoretical results for ferromagnetic instability in interacting $2 \mathrm{D}$ and $3 \mathrm{D}$ quantum Coulomb systems; we conclude in $\nabla$ with a discussion of experimental implications and related open questions.

\section{BACKGROUND}

The possibility of a density driven ferromagnetic transition in an interacting electron system was first suggested by Bloch ${ }^{39}$ more than 75 years ago. Bloch's basic idea, essentially a Hartree-Fock mean field theory, remains fundamentally valid even today. The idea is that at high density the electron system would be paramagnetic in order to optimize the kinetic energy cost (which is high in a high-density quantum fermionic system) whereas at low density the system should spontaneously spin-polarize itself into a ferromagnetic ground state in order to optimize the exchange energy arising from the Pauli principle and Coulomb interaction. For an electron gas (in a positive jellium background) it is a straightforward exercise to write down the total HartreeFock energy per-particle as a sum of the non-interacting kinetic energy and the (Fock) exchange energy due to unscreened Coulomb interaction at $T=0$ as

$$
\begin{aligned}
\frac{E}{n}=\frac{E_{K E}}{n}+\frac{E_{e x}}{n} & =\frac{0.55}{r_{s}^{2}}\left[(1+\zeta)^{5 / 3}+(1-\zeta)^{5 / 3}\right] \\
& -\frac{0.23}{r_{s}}\left[(1+\zeta)^{4 / 3}+(1-\zeta)^{4 / 3}\right](1)
\end{aligned}
$$

for 3D system, and

$$
\frac{E}{n}=\frac{0.50}{r_{s}^{2}}(1+\zeta)^{2}-\frac{0.30}{r_{s}}\left[(1+\zeta)^{3 / 2}+(1-\zeta)^{3 / 2}\right]
$$

for $2 \mathrm{D}$ systems, where $n=n_{\uparrow}+n_{\downarrow}$ is the total number density of electrons; $\zeta=\left(n_{\uparrow}-n_{\downarrow}\right) / n$ is their spinpolarization (or magnetism) density; $a_{B}^{3} n=\left(\frac{4}{3} \pi r_{s}^{3}\right)^{-1}$ and $a_{B}^{2} n=\left(\pi r_{s}^{2}\right)^{-1}$ define the dimensionless interaction parameters $r_{s}$ in $2 \mathrm{D}$ and $3 \mathrm{D}$ respectively (with $a_{B}=\hbar^{2} /\left(m e^{2}\right)$, the Bohr radius); and the energy is measured in Rydberg units (i.e. $e^{2} / a_{B}$ ). It is easy to see that the above Hartree-Fock energy expressions lead to a first-order ferromagnetic transition (the "Block ferromagnetism") at $r_{s}=r_{B}$ where $r_{B} \simeq 5.45(3 \mathrm{D})$ and $r_{B} \simeq 2(2 \mathrm{D})$, i.e. $E(\zeta=1)$ ferromagnetic state is lower (higher) in energy than $E(\zeta=0)$ paramagnetic state for $r_{s}>(<) r_{B}$. We refer to such energy-difference-driven abrupt (first order) transition as Bloch ferromagnetism in the rest of this paper.
The Stoner ferromagnetic instability $\underline{\underline{40}}$ refers to the divergence of the spin susceptibility and hence a secondorder continuous magnetic phase transition from a paramagnetic $\left(r_{s}<r_{S t}\right)$ weak-coupling side to a ferromagnetic side $\left(r_{s}>r_{S t}\right)$. The simplest model to consider is, following Stoner's original work, a zero-range delta-function like interaction of strength ' $I$ ' (a constant in momentum space) between the electrons, leading to an interacting static long-wavelength spin susceptibility (in the dynamical Hartree-Fock approximation) given by $\chi^{*} / \chi=\left(1-D_{0} I\right)^{-1}$, where $D_{0} \equiv D\left(E_{F}\right)$ is the electronic density of states at the Fermi energy. This immediately leads to the Stoner criterion for ferromagnetic instability defined by a divergent $\chi^{*} / \chi$ when $1-D_{0} I=0$. Since the $2 \mathrm{D}$ density of states is a density-independent constant, this instability criterion does not lead to a meaningful condition in 2D unless we arbitrarily define $I$ to be the Coulomb interaction strength at Fermi wavevector, whence the Stoner instability criterion leads to unphysically low $r_{s}$ values for the ferromagnetic instability given by $r_{S t} \simeq 0.7(2 \mathrm{D})$ and $r_{S t} \simeq 1.5(3 \mathrm{D})$, which are absurdly small $r_{s}$-values and are unrealistic. Of course, in real electron systems the electron-electron interaction is the long-range Coulomb interaction, and therefore the simple Stoner instability criterion, defined by $D_{0} I=1$ where $I$ is an effective short-range interaction strength, is inapplicable. But the basic idea of the ferromagnetic Stoner instability, defined by a continuous divergence of $\chi^{*}\left(r_{s}\right)$ as $r_{s}$ is increased, still applied. We refer to the ferromagnetic transition defined or characterized by a divergence of the interacting susceptibility as the Stoner instability.

In real electron liquids, the exchange-only HartreeFock approximation considered above for the Bloch instability is inadequate because correlation effects (i.e. energy contributions beyond Hartree-Fock) are known to be extremely important, and must be included in the energetic considerations. Similarly, the interacting susceptibility must be calculated for the real Coulomb interaction in the system, not for a hypothetical zero-range interaction, in order to obtain a better estimate of the Stoner instability criterion. In the rest of this paper we consider both Bloch and Stoner ferromagnetic instabilities using better many-body approximations for Coulomb electron liquids, namely RPA.

\section{THEORY}

In this work we follow the notation of Refs. 36 37 41 . Within $\mathrm{RPA}^{36,37.38,41,42.43,44}$, the Coulomb contribution to the ground state energy of a jellium electron system with long-range Coulomb interaction can be denoted by the Feynman diagrams shown in Fig. 17A. The quasiparticle energy is then obtained by $E_{\mathbf{k}}=\delta E_{G} / \delta n_{\mathbf{k}}$, where $n_{\mathbf{k}}$ is the distribution function at momentum $\mathbf{k}$. The second order derivative of the total ground state energy is referred to as Landau's interaction function: 
(A)

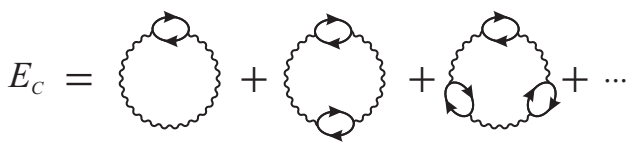

(B)

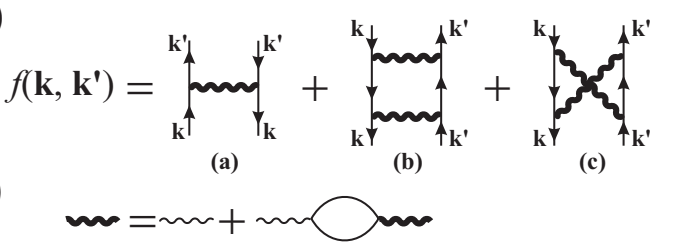

FIG. 1: The RPA Feynman diagram for: (A) the Coulomb interaction contribution to the ground state energy; (B) Landau's interaction function; (C) Dynamically screened interaction. The circles are polarization bubbles, the thin wiggly lines are the bare Coulomb interaction, and the solid lines the noninteracting electron Green's function.

$f\left(\mathbf{k}, \mathbf{k}^{\prime}\right)=\delta^{2} E_{G} / \delta n_{\mathbf{k}} \delta n_{\mathbf{k}^{\prime}}$, represented by the Feynman diagram shown in Fig. 1B. Graphically, taking the $n_{\mathbf{k}}$ variational derivative of a quantity simply means that one cuts one solid line of the Feynman diagram and takes the external momentum and frequency to be on-shell (i.e. $\omega=\mathbf{k}^{2} / 2 m-E_{F}$ with $m$ the band electron mass and $E_{F}$ the Fermi energy). We emphasize that the RPA as shown in Fig. 10 1necessarily implies that this on-shell selfenergy approximation is used for calculating the quasiparticle energy dispersion $E_{\mathbf{k}}$ and Landau's interaction function $f\left(\mathbf{k}, \mathbf{k}^{\prime}\right)$ since all energy and momenta in Fig. [1 correspond to the noninteracting system. Thus RPA selfenergy approximation necessarily implies an on-shell approximation (Fig. TA) as emphasized by Rice $\frac{42}{2}$ a long time ago. Solving the full Dyson equation using the offshell renormalized energy, as is sometimes done in the literature, would be completely inconsistent within RPA as it would incorrectly mix various perturbative orders.

Following Hubbard's notation ${ }^{43}$, the ground state energy per particle $E_{G} / N$ with $N$ the particle number can be written as $E_{G} / N=E_{K} / N-v(0) / 2+E_{C} / N$, where $E_{K}$ is the kinetic energy, $v(0)=\int v_{q} d^{d} q /(2 \pi)^{d}$ is the interaction energy at zero separation with the bare Coulomb interaction $v_{q}=2 \pi e^{2} / q$ for $2 \mathrm{D}$ and $v_{q}=$ $4 \pi e^{2} / q^{2}$ for $3 \mathrm{D}$, and $E_{C}$ is the Coulomb contribution to the ground state energy (both exchange and correlation) which can be denoted as in Fig. 11A. Note that the singularities in $v(0) / 2$ and $E_{C} / N$ cancel out with each other. In the $2 \mathrm{D}$ system, it is easy to show ${ }^{43}$ that RPA leads to

$$
\begin{aligned}
\frac{E_{G}}{N} & =\frac{E_{F}}{2}+\frac{E_{e x}}{N} \\
& +\frac{16 E_{F}}{g_{s} \pi} \int_{0}^{\infty} x d x \int d u[\ln \epsilon(x, u)-\epsilon(x, u)],
\end{aligned}
$$

where $\epsilon(q, \omega)$ is the dynamical dielectric function, $g_{s}$ is the spin degeneracy $\left(g_{s}=2\right.$ for paramagnetic states and $g_{s}=1$ for fully spin-polarized state), $E_{\text {ex }}$ is the exchange part of the ground state energy. Note that in Eq. (3) we subtracted a term $\epsilon(q, \omega)$ from $\ln [\epsilon(q, \omega)]$ in order to handle the ultraviolet divergence in the integration. Similarly for $3 \mathrm{D}$ we have

$$
\begin{aligned}
\frac{E_{G}}{N} & =\frac{3}{5} E_{F}+\frac{E_{e x}}{N} \\
& +\frac{48 E_{F}}{g_{s} \pi} \int_{0}^{\infty} x^{2} d x \int d u[\ln \epsilon(x, u)-\epsilon(x, u)] .
\end{aligned}
$$

It is convenient to convert all the expressions in terms of the dimensionless units $r_{s}$. The definition of $r_{s}$ is as $r_{s}=1 /\left(\alpha k_{F} a_{B}\right)$ where $k_{F}$ is the Fermi momentum and $a_{B}=1 /\left(m e^{2}\right)$ is the Bohr radius. In $2 \mathrm{D} \alpha=\sqrt{g_{s}} / 2$ and in $3 \mathrm{D} \alpha=\left(2 g_{s} / 9 \pi\right)^{1 / 3}$. Also we choose $\hbar=1$ throughout, which makes wavevector and momentum (as well as energy and frequency) equivalent. In these notations, it is easy to show that $E_{e x} / N=-8 \alpha r_{s} /(3 \pi) E_{F}$ for $2 \mathrm{D}$, and $E_{e x} / N=-3 \alpha r_{s} /(2 \pi) E_{F}$ for 3D. In the actual calculation, the integration in Eqs. (3) and (4) can be performed on either real or imaginary axis. By examining the $r_{s}$ and $g_{s}$ dependence of the ground state energy, we study the Bloch magnetic instability of the electron system. The integrals in Eqs. (3) and (4) are the correlation contributions not included in our Hartree-Fock considerations of Bloch ferromagnetic in Sec. III Note that $g_{s}=1(2)$ corresponds to $\zeta=1(0)$ in Sec. III (a)

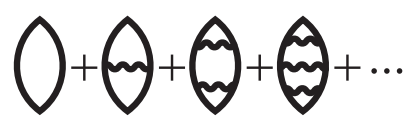

(c)

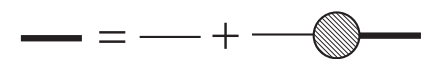

(e)

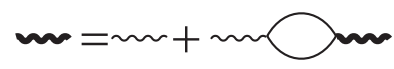

(b)

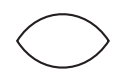

(d)

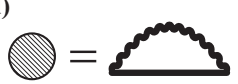

(f)

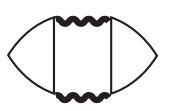

FIG. 2: (a) The ladder-bubble series for the interacting susceptibility with the bold straight line the interacting Green's function and the bold wavy line the dynamically screened interaction; (b) the noninteracting susceptibility; (c) the Dyson's equation for the interacting Green's function in terms of the noninteracting Green's function and the self-energy; (d) the self-energy in the leading-order expansion in the dynamical screening; (e) the Dyson's equation for the dynamically screened interaction in terms of the bare Coulomb interaction (thin wavy lines) and the polarization bubble; (f) a charge fluctuation diagram which does not contribute to spin susceptibility.

We investigate the Stoner instability by calculating the magnetic susceptibility $\chi^{*}$ within RPA which is represented by the Feynman diagram showed in Fig. 22 Direct calculation of these diagrams turns out to be difficult for the long ranged Coulomb interaction. However, at $T=0$, Landau showed that $\chi^{*}$ can be equivalently expressed as the following equation ${ }^{42}$ :

$$
\frac{\chi}{\chi^{*}}=\frac{m}{m^{*}}+\varpi \int f_{e}(\theta) d o,
$$


where $\chi$ is the Pauli spin susceptibility, $f_{e}(\theta)=f_{e}\left(\mathbf{k}, \mathbf{k}^{\prime}\right)$ with $\mathbf{k}$ and $\mathbf{k}^{\prime}$ on-shell; $\mathbf{k}^{2} / 2 m=\mathbf{k}^{\prime 2} / 2 m\left(=E_{F}\right)$ is the exchange Landau's interaction function, $\theta$ is the angle between $\mathbf{k}$ and $\mathbf{k}^{\prime}$, do the element of solid angle along $\mathbf{k}^{\prime}$ times $\cos \theta$ in $3 \mathrm{D}$ and $d \theta$ in $2 \mathrm{D}$, and $\varpi=1 /(2 \pi)^{2}$ in $2 \mathrm{D}$ and $\varpi=k_{F} /(2 \pi)^{3}$ in 3D. Similarly, the Landau theory expression for the effective mass $m^{*}$ is ${ }^{42}$

$$
\frac{m}{m^{*}}=1-\varpi \int f(\theta) d o .
$$

In Eq. (5), $f_{e}(\theta)$ is the exchange part of the Landau's interaction function, which is represented by Fig. 1B B(a). This spin independent term is responsible for the difference between the ratio $\chi / \chi^{*}$ and $m / m^{*} \underline{42}$. An equivalent, and easier way to derive the effective mass is through calculating quasiparticle self-energy and obtaining its momentum derivative. The self-energy within RPA can be written as ${ }^{36}$

$$
\Sigma(\mathbf{k}, \omega)=-\int \frac{d^{d} q}{(2 \pi)^{d}} \int \frac{d \nu}{2 \pi i} \frac{v_{q}}{\epsilon(\mathbf{q}, \nu)} G_{0}(\mathbf{q}+\mathbf{k}, \nu+\omega),
$$

where $d=2$ or 3 is the dimension of the system, and

$$
G_{0}(\mathbf{k}, \omega)=\frac{1-n_{F}\left(\xi_{\mathbf{k}}\right)}{\omega-\xi_{\mathbf{k}}+i 0^{+}}+\frac{n_{F}\left(\xi_{\mathbf{k}}\right)}{\omega-\xi_{\mathbf{k}}-i 0^{+}}
$$

is the bare Green's function, where $n_{F}$ is the Fermi distribution function, $\xi_{\mathbf{k}}=k^{2} /(2 m)-E_{F}$. It is shown 37 that the integration along real axis in the expression of self-energy (Eq. (7)) can be deformed onto imaginary axis, which avoids the singularities along the real axis and makes the integration easier. The contour deformation also breaks the expression of self-energy into separate terms that correspond respectively to contributions from the spin-dependent and spin-independent part of the Landau's interaction function shown in Fig. 1] and is very useful for us to derive the expression for susceptibility as shown below. The expression of the real part of the self-energy can then be written as

$$
\begin{aligned}
\operatorname{Re} & \Sigma(\mathbf{k}, \omega)=-\int \frac{d^{d} q}{(2 \pi)^{d}} v_{q} \Theta\left(2 m \omega+k_{F}^{2}-|\mathbf{q}-\mathbf{k}|^{2}\right) \\
+ & \int \frac{d^{d} q}{(2 \pi)^{d}} v_{q} \operatorname{Re} \frac{1}{\epsilon\left(\mathbf{q}, \xi_{\mathbf{q}-\mathbf{k}}-\omega\right)} \\
& \cdot\left[\Theta\left(2 m \omega+k_{F}^{2}-|\mathbf{q}-\mathbf{k}|^{2}\right)-\Theta\left(k_{F}^{2}-|\mathbf{q}-\mathbf{k}|^{2}\right)\right] \\
- & \int \frac{d^{d} q}{(2 \pi)^{d}} \int \frac{d \nu}{2 \pi} v_{q}\left[\frac{1}{\epsilon(\mathbf{q}, i \nu)}-1\right] \frac{1}{i \nu+\omega-\xi_{\mathbf{q}+\mathbf{k}}} \cdot(9)
\end{aligned}
$$

The effective mass is derived from the expression of the real part of the quasiparticle self-energy by $m / m^{*}=$ $1+\left.\left(m / k_{F}\right) \frac{d}{d k} \operatorname{Re} \Sigma\left(\mathbf{k}, \xi_{\mathbf{k}}\right)\right|_{k=k_{F}} \frac{42}{}$. Combining this with Eq. (5), we have

$$
\frac{\chi}{\chi^{*}}=1+\varpi \int f_{e}(\theta) d o+\left.\frac{m}{k_{F}} \frac{d}{d k} \operatorname{Re} \Sigma\left(\mathbf{k}, \xi_{\mathbf{k}}\right)\right|_{k=k_{F}} .
$$

It is not difficult to show that the second term of Eq. (9) accounts for the contribution from the spinindependent exchange Landau's interaction function $f_{e}\left(\mathbf{k}, \mathbf{k}^{\prime}\right)($ Fig. 1 $\mathrm{B}(\mathrm{a}))$, and therefore the term $\varpi \int f_{e}(\theta) d o$ in Eq. (10) exactly cancels the momentum derivative of the second term in the self-energy Eq. (9). Hence the expression of $\chi / \chi^{*}$ only contains contributions from the $k$ derivatives of the first and third term in Eq. (9). After converting all the expressions in terms of the dimensionless parameter $r_{s}$, and using $2 k_{F}, 4 E_{F}, 2 m$ as the momentum, energy, and mass units, we obtain the expression for the $2 \mathrm{D}$ magnetic susceptibility as

$$
\begin{aligned}
\frac{\chi}{\chi^{*}}= & -\frac{2 \alpha r_{s}}{\pi}+\frac{\sqrt{2} \alpha r_{s}}{\pi} \int_{0}^{\infty} x^{2} d x \int_{0}^{\infty} d u\left[\frac{1}{\epsilon(x, i u)}-1\right] \\
& \times[A \sqrt{1+A / R}-B \sqrt{1-A / R}] R^{-5 / 2}
\end{aligned}
$$

where $A=x^{4}-x^{2}-u, B=2 x u, R=\sqrt{A^{2}+B^{2}}$. Similarly for $3 \mathrm{D}$ we have

$$
\begin{aligned}
\frac{\chi}{\chi^{*}}=-\frac{\alpha r_{s}}{\pi} & +\frac{\alpha r_{s}}{2 \pi^{2}} \int_{0}^{\infty} d x \int_{0}^{\infty} d u\left[\frac{1}{\epsilon(x, i u)}-1\right] \\
& \times[\ln (F / G)-2 C / F+2 D / G]
\end{aligned}
$$

where $C=1-q, D=1+q, F=C^{2}+u^{2}, G=D^{2}+u^{2}$. Note that in the expressions of the dielectric function $\epsilon(x, u), x=q /\left(2 k_{F}\right)$ and $u=\omega /\left(4 E_{F}\right)$.

\section{RESULTS}

In Fig. [3 we present the calculated magnetic susceptibility as a function of $r_{s}$ for both 2D and 3D systems, together with the calculated $g$-factor $g^{*}=\chi^{*} / m^{*}$ and the effective mass $m^{*}$. It is clear from Fig. 3 that both $2 \mathrm{D}$ and 3D systems experience Stoner ferromagnetic instabilities, characterized by the divergence of magnetic susceptibility as the density decreases. It is important to note that this Stoner instability (i.e. divergence of $\chi^{*}$ ) does not arise from an effective mass divergence since the $m^{*}$ divergence happens at much lower densities. In other words, $g^{*}$ and $m^{*}$ both diverge, with the divergence of $g^{*}$ occurring at a lower $r_{s}$ value. For the $2 \mathrm{D}$ system, $\chi^{*}$ and $g^{*}$ diverges at $r_{s} \sim 7.3$ while $m^{*}$ diverges at $r_{s} \sim 18.1$. For the 3D system, $\chi^{*}$ and $g^{*}$ diverge at $r_{s} \sim 18.7$ while $m^{*}$ diverges at $r_{s} \sim 49.9$.

We emphasize that both $m^{*}$ and $g^{*}$ divergences actually happen independently and are completely unrelated phenomena. On the other hand, it is also worth mentioning that the $g^{*}$ divergence does have some quantitative effect on the $m^{*}$ divergence. After $g^{*}$ diverges, the system becomes a ferromagnetic liquid, and the momentum distribution is different and the Fermi energy increases. This change results in a small increase in the critical $r_{s}$ value where $m^{*}$ diverges. In fact for $g_{s}=2$ paramagnetic systems, $m^{*}$ diverges at 16.1 for $2 \mathrm{D}$ and 47.8 for $3 \mathrm{D}^{36}$, in contrast to $g_{s}=1$ case where $m^{*}$ diverges at 18.1 for 

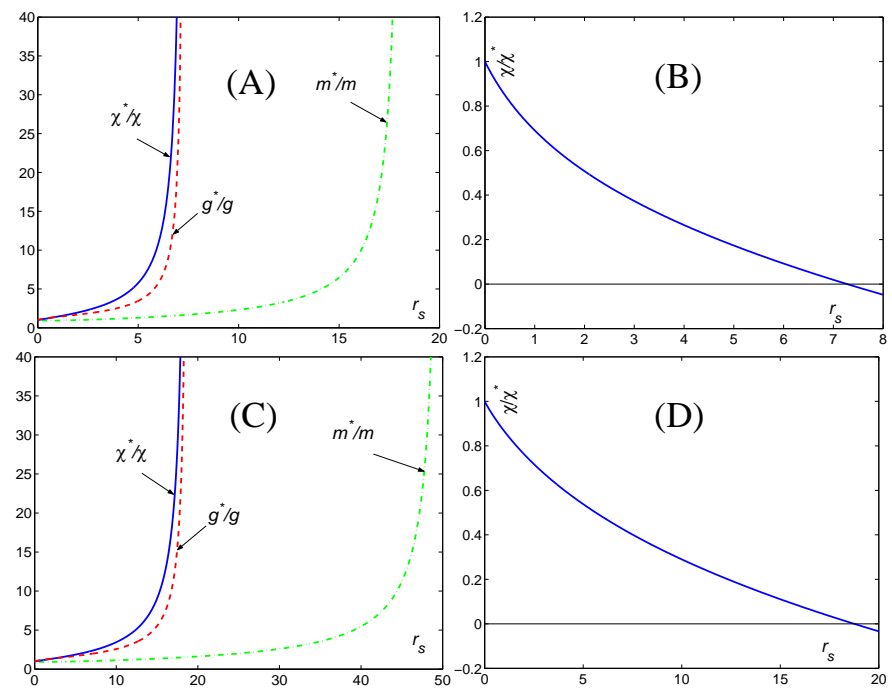

FIG. 3: (A) and (C): Calculated renormalized spin susceptibility $\chi^{*} / \chi$, effective mass $m^{*} / m$ and $g$-factor $g^{*} / g$. For $2 \mathrm{D}$ system, $\chi^{*}$ and $g^{*}$ diverge at $r_{s} \sim 7.3$ while $m^{*}$ diverges at $r_{s} \sim 18.1$. For 3D system, $\chi^{*}$ and $g^{*}$ diverges at $r_{s} \sim 18.7$ while $m^{*}$ diverges at $r_{s} \sim 49.9$. (B) and (D): inverse susceptibility shows $\chi^{*}$ diverges at $r_{s} \sim 7.3$ for $2 \mathrm{D}$ and 18.7 for $3 \mathrm{D}$ systems. Note that the $\chi^{*}$ and $g^{*}$ are calculated for paramagnetic systems $\left(g_{s}=2\right)$ while $m^{*}$ are for ferromagnetic systems $\left(g_{s}=1\right)$.
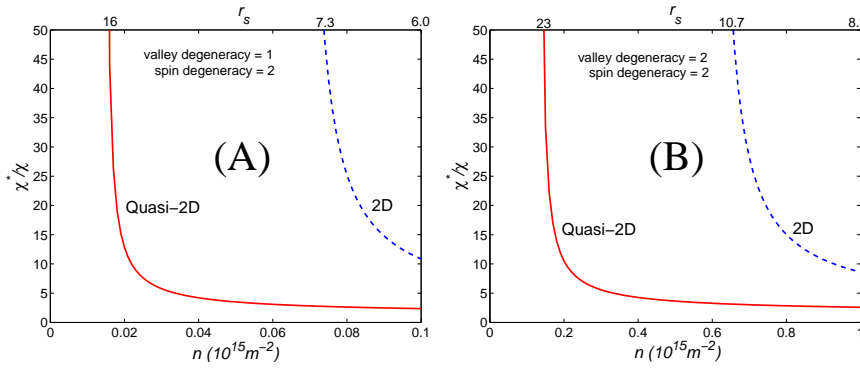

FIG. 4: Quasi-2D effects on magnetic susceptibility divergence. (A): GaAs quantum well system. (B): Si-inversion layer.

$2 \mathrm{D}$ and 49.9 for $3 \mathrm{D}$. But this effect is rather small and is of no particular significance.

In real experimental systems, the value of $r_{s}$ at which $\chi^{*}$ diverges should be influenced by many factors (even within our RPA many-body approximation scheme). Here we consider the valley degeneracy $g_{v}$ and the finite width effect on the magnetic susceptibility divergence in semiconductor-based realistic $2 \mathrm{D}$ electron systems. The effect of $g_{v}$ is exactly the same as the effect of $g_{s}$ on the system, and therefore can be easily incorporated. For the finite width effect, we introduce a form factor to the Coulomb interaction, following the standard procedure described in detail in Refs. 4546 . Using appropriate semiconductor parameters, we obtain the susceptibility in GaAs quantum wells and Si-inversion layers, plotted in Fig. 4 It is clear from Fig. 4 that multi-valley degen- eracy and finite width both suppress the divergence of the susceptibility renormalization, and make the critical $r_{s}$ value for $\chi^{*}$ divergence considerably larger than the strict $2 \mathrm{D}$ results.
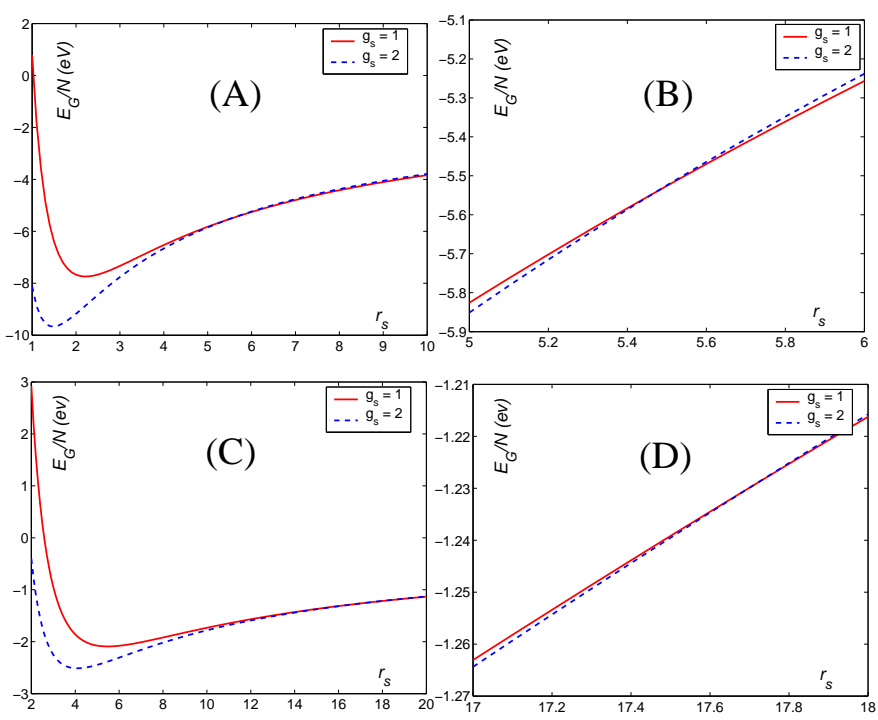

FIG. 5: (A) and (C): the RPA ground state energy per particle for polarized $\left(g_{s}=1\right)$ unpolarized $\left(g_{s}=2\right)$ cases as a function of $r_{s}$ for 2D and 3D systems, calculated using Eq. (3) and Eq. (4). (B) and (D): details around the magnetic instability at $r_{s} \sim 5.5$ for $2 \mathrm{D}$ and 17.8 for $3 \mathrm{D}$ systems.

The magnetic susceptibility is a thermodynamic Fermisurface property. As mentioned before, another way of studying the magnetic instability is to compare the ground state energy of the system for polarized and unpolarized states at different electron densities. This is the Bloch ferromagnetism discussed in Sec. III Our results (Fig. [5) of RPA ground state energy for fully polarized and non-polarized electron states (using Eqs. (3) and (4) in both 2D and 3D electron systems show similar characteristic. When $r_{s}$ is very small (or electron densities high), both systems prefer non-polarized paramagnetic states. As $r_{s}$ increases to a certain critical value $\left(r_{s} \sim 5.5\right.$ for $2 \mathrm{D}$ and $r_{s} \sim 17.8$ for $\left.3 \mathrm{D}\right)$ the ground state energy for the fully polarized electron state actually becomes lower than the non-polarized states. This clearly indicates that the system undergoes a Bloch type ferromagnetic instability due to the Coulomb electron-electron interaction in a low density 2D electron systems. Note that the critical $r_{s}$ for the Bloch instability is substantially higher in the RPA theory (increasing from 2 to 5.5 in $2 \mathrm{D}$ and 5.45 to 17.8 in $3 \mathrm{D}$ ) than in the Hartree Fock theory due to the inclusion of correlation energy.

Comparing Figs. 3 and $[5$ we conclude that, at least within our well-defined RPA ring-diagram many-body approximation scheme, the sequence of instabilities (as density decreases) the theory predicts for both $2 \mathrm{D}$ and 3D electron liquids is the following: Bloch ferromagnetism (i.e. an abrupt first order magnetic transition) at $r_{s}=5.5(2 \mathrm{D}) ; 17.8(3 \mathrm{D})$; Stoner ferromagnetism char- 
acterized by a continuous divergence (i.e. a second order magnetic transition) of the interacting $g$-factor and of the susceptibility at $r_{s}=7.3(2 \mathrm{D}), 18.7(3 \mathrm{D})$; the dispersion instability associated with the continuous divergence of the quasiparticle effective mass at $r_{s}=18.1$ (2D), 49.9 (3D). Of course, comparing first and second order transitions is not particularly meaningful since their origins are fundamentally different, and a first order transition may always preempt a second order transition as seems to happen in Coulomb electron liquids with Bloch ferromagnetism always happening (both in $2 \mathrm{D}$ and $3 \mathrm{D}$ ) at a slightly lower $r_{s}$ values (5.5 versus 7.3 in $2 \mathrm{D}$, and 17.8 versus 18.7 in $3 \mathrm{D}$ ) although the difference in the critical $r_{s}$ values for the two transitions (less than $10 \%$ in 3D and about $25 \%$ in $2 \mathrm{D}$ ) is sufficiently small so that both Bloch and Stoner ferromagnetism remain of experimental interest.

We also note that, by definition, $\chi^{*} \equiv g^{*} m^{*}$ (and $\chi=g m)$, and therefore the divergence of the interacting susceptibility could be caused either by a divergence $g$ factor or a diverging effective mass. This issue has been much discussed and debated $1,2,3,10,11,12.13$ in the recent experimental literature on 2D semiconductor-based electron systems, where low-density divergence of both $2 \mathrm{D}$ susceptibility and effective mass has been reported. All we can say is that our theoretical results are only consistent with the susceptibility divergence as arising from the divergence of the interacting $g$-factor, not the effective mass, since the $g$-factor divergence occurs at much lower $r_{s}$-values, $r_{s} \sim 7.3(18.7)$ in $2 \mathrm{D}(3 \mathrm{D})$ for $g^{*} / g$ divergence versus $r_{s} \sim 18.1(49.9)$ in $2 \mathrm{D}(3 \mathrm{D})$ for $m^{*} / m$ divergence. We add that in realistic quasi-2D semiconductor system (our Fig. (4) the susceptibility (as well as effective mass) divergence occurs at substantially higher $r_{s}$ values due to the considerable softening of the Coulomb interaction from its strict $2 \mathrm{D}$ form due to the finite-width effect.

\section{DISCUSSION}

Both Bloch and Stoner instabilities imply that 2D and $3 \mathrm{D}$ electron systems interacting via the long range Coulomb interaction undergo a $T=0$ ferromagnetic quantum phase transition from a high-density paramagnetic state to a low-density ferromagnetic state either through a first order (Bloch) transition or a second-order (Stoner) transition (with a continuous divergent susceptibility) at a critical $r_{s}$-value. Given that the critical $r_{s}$ value(s) for the transition(s) we obtain within our RPA many-body theory is rather large (i.e. $r_{s} \gg 1$ ), we do not expect our predicted $r_{s}$ parameter for ferromagnetic transitions in 2D and 3D electron systems to be reliable. But the basic trends, such as the sequence of instabilities (i.e. Bloch followed by Stoner followed by the dispersion instability with diverging mass as the density is lowered) or the suppression of the transition to much lower densities in quasi-2D systems, should be valid, in general. Indeed quantum phase transitions predicted by RPA (or for that matter even by the simpler Hartree-Fock approximation) are always found to occur in the numerical quantum Monte Carlo (QMC) simulation albeit at higher $r_{s}$ values. This is certainly true for electron liquid ferromagnetic instabilities. For 3D system, QMC simulation predicts that the system undergoes a possibly second-order phase transition and electron spins become partially polarized at $r_{s} \sim 60$ by Ref. 47 or $r_{s} \sim 15-25$ by Ref. 17, and as $r_{s}$ increase to $r_{s} \sim 100$ by Ref. 47 or $r_{s} \sim 35-45$ by Ref. 17, the system becomes a fully polarized ferromagnetic state. For 2D case, QMC simulation predicts $\frac{48}{2}$ that as density approaches $r_{s} \sim 25$, the system undergoes a first-order transition into a fully spin-polarized ferromagnetic state. Theoretically it is possible to obtain the spin susceptibility through calculating the second derivative of the ground state energy with respect to $\zeta$ at $\zeta=0$. However, in reality too much error is introduced when obtaining the susceptibility this way in QMC simulations, and therefore it is difficult to ascertain the order of the ferromagnetic transition in QMC numerical simulations - our RPA theory predicts the first-order Bloch instability to occur first as the density is being lowered. Another thing to be noticed about QMC simulation results is that the ferromagnetic transition $r_{s}$ value predicted by different groups differs by a large factor from each other (see, for example, Ref. 17 and Ref. 47), which shows the large amount of error introduced by such simulations due to the small energy difference between spin polarized states and spin unpolarized states (the two density dependent energy curves are almost parallel when they cross each other) and different choices of trial wave functions.

Much has been written about the validity of the RPA many-body approximation at low carrier densities $\left(r_{s}>\right.$ 1). We have little to add to this issue beyond the detailed discussion we already provided in our recent publications ${ }^{36.41}$. We want to emphasize that, although RPA is exact in the $r_{s} \rightarrow 0$ limit, it is by no means a theory based on an $r_{s}$ expansion - it is a self-consistent field theory based on an expansion in the dynamically screened interaction which should be qualitatively valid for all $r_{s}$ below the Wigner crystallization of the electron liquid. In fact, RPA is found to be quantitatively valid in $3 \mathrm{D}$ systems ${ }^{42}$ at metallic densities $\left(r_{s} \sim 3-6\right)$ and in $2 \mathrm{D}$ systems for $r_{s}$ up to $10-15$ where comparison with experiment has been carried out $\underline{49}$. Often the error in the calculation arising from other effects (e.g. finite temperature ${ }^{50}$, finite quasi-2D width, band structure, etc.) turn out to be larger than that included in the RPA approximation, and therefore improvement beyond RPA (short of a full-fledged QMC calculation) becomes meaningless. One can try to "improve" upon RPA by including local field corrections to the dynamical electron polarizability (i.e. bare bubble of RPA) which, in some crude manner, simulates the incorporation of higher-order vertex corrections in the theory. But such local field corrections are uncontrolled, and probably inconsistent, since many diagrams in the same order are typically left out. We are therefore unconvinced that the inclusion of local field cor- 
rections in the theory is necessarily an improvement on RPA. The great conceptual advantage of RPA is that it is a well-defined approximation that is both highly physically motivated (i.e. dynamical screening) and theoretically exact in the high-density $\left(r_{s} \rightarrow 0\right)$ limit. Attempted improvement upon RPA through the arbitrary inclusion of local field correction may neither be theoretically justifiable nor more reliable. Keeping these caveats in mind we have carried out our exchange instability calculations using the Hubbard local field corrections ${ }^{43}$, and we find no qualitative changes from the RPA results presented in this paper. The critical $r_{s}$ values for the occurrence of the ferromagnetic instability change somewhat in the presence of the local field correction, but this is a result without any significance since the precise values of critical $r_{s}$ are expected to be not particularly accurate in any of these theories. The fact that the basic qualitative conclusions about the various instabilities do not change in the presence of local field corrections demonstrates the qualitative robustness of our RPA-based results. Another point to note is that the fact that RPA predicts the existence of divergence of certain physical quantities at certain critical densities does not necessarily imply that RPA or Fermi liquid theory fails at that density. For example, RPA predicts the divergence and then negative values for the compressibility ${ }^{38}$ at densities higher than ferromagnetic transition densities, and negative compressibility has indeed been observed in experiments 51 on $2 \mathrm{D}$ electron systems as predicted by RPA calculations. Of course the total compressibility of a system cannot be negative, but just the electronic part of the compressibility can be negative as predicted by RPA and as is routinely observed in $2 \mathrm{D}$ electron systems for $r_{s}>3$. There can be no doubt that if 3D electron systems with large enough $r_{s}$ values $\left(r_{s}>6\right.$ according to RPA $\left.{ }^{38}\right)$ are found they would routinely have negative electronic compressibility as well! It is certainly true that RPA becomes a progressively poorer approximation as density decreases and perhaps detailed QMC calculations should be carried out to test the validity of our RPA-based predictions presented in this paper.

In discussing possible experimental implications of our results, we note the great recent experimental interest in the literature on the possibility of a densitydriven ferromagnetic transition in semiconductor-based 2D carrier systems where very low carrier densities $\left(r_{s} \lesssim 10\right)$ can be achieved in rather high-quality samples $1,2.3 .4,5.6,7,8,9,10,11,12,13,14$. There are recent experimental claims ${ }^{1.2 .3 .12}$ of the observation of a low density susceptibility divergence in Si MOSFETs for $r_{s} \sim 7-10$. Although the experimental susceptibility behavior as a function of density (or, $r_{s}$ ) looks similar to our theoretical results in Figs. 3 and 4 we are skeptical about the significance of this agreement. There are several reasons for our skepticism. First, the experimental claimed susceptibility divergence occurs at far too high a density $\left(r_{s} \sim 7\right)$ compared with the theory where we find the RPA susceptibility divergence in realistic Si MOSFETs
(Fig. (4) to be occurring at $r_{s} \approx 23$. This RPA predictions for critical $r_{s}(\sim 23)$ is most likely the lower bound - any real susceptibility divergence is expected to occur at higher $r_{s}$ values $\left(r_{s}>23\right)$. Second, the experimental divergence of $\chi^{*} / \chi \equiv g^{*} m^{*} / g m$ has been claimed ${ }^{1.2 .3}$ to be arising from an effective mass divergence, not the $g$-factor divergence as we find in our theory. Our theoretical effective mass divergence, in fact, occurs at a critical $r_{s}$ more than twice as large as the corresponding $\chi^{*}$ critical $r_{s}$. In fact, our quasi-2D effective mass divergence ${ }^{36.37}$ occurs for $r_{s}>40$ ! Third, there has been no experimental evidence for the existence of a low-density ferromagnetic phase such as hysteresis, remanence, etc. If there is indeed a ferromagnetic transition, one should be able to observe ferromagnetic behavior at densities below the ferromagnetic critical density (i.e. for $r_{s}$ values larger than the point of $\chi^{*}$ divergence). No such direct ferromagnetic behavior has ever been observed in a lowdensity 2D system casting serious doubts on the claims of the observation of a $2 \mathrm{D}$ ferromagnetic transition. A very recent extremely careful and detailed measurement of $2 \mathrm{D}$ susceptibility in a high-mobility n-GaAs system ${ }^{9}$ finds no divergence in $\chi^{*} / \chi$ up to $r_{s} \approx 12$, calling into question the earlier claims of susceptibility divergence in Si MOSFETs at lower $r_{s}$ values. In addition, a direct thermodynamic measurement $\underline{\underline{4}}$ of $2 \mathrm{D}$ susceptibility in $\mathrm{Si}$ MOSFETs also does not find a ferromagnetic instability. What is clear is the observed strong enhancement of $\chi^{*} / \chi$ (and $m^{*} / m$ ) as a function of increasing $r_{s}$ which is consistent with our theoretical findings. But the actual existence of a low-density electron liquid ferromagnetic transition has not be established experimentally in our opinion.

Finally we discuss some of the earlier literature that is of relevance to our work. Our calculation of the ground state energy for polarized and unpolarized states partially confirms the numerical results of Rajagopal et. $a l^{52}$, who also considered the possibility of partially polarized states, and found that for certain range of electron densities, the system prefers a partially polarized ferromagnetic state in the 3D system. Similar results were also derived using the Quantum Monte Carlo $\operatorname{method}^{17.47}$. In this paper we did not present our calculation results (which confirms the results of Ref ${ }^{52}$ ) for the Bloch instability associated with the partial spinpolarization because this will not help our understandings of the relation between Stoner, Bloch, and dispersion instabilities, which is the main purpose of this paper. In 3D, partially spin polarized states is preferred energetically for the density region of $r_{s}$ between $\sim 14$ and $\sim 18$, which is right before the fully polarized ferromagnetic region as the density is decreased 52 . This suggests that our understanding of the relation between the three kinds of instabilities will not be affected by the consideration of partially spin-polarized states. In 2D systems, partial spin-polarization does not occur ${ }^{17.47 .52}$, and thus this issue does not arise at all for our 2D calculations, which is the main focus of our work. For the 
magnetic susceptibility, there have been earlier RPA calculations in $2 \mathrm{D}^{29.30 .31 .53 .54}$ and $3 \mathrm{D}^{28}$, and QMC calculations in $2 \mathrm{D}^{48}$. Only Shastry ${ }^{28}$ predicted a susceptibility divergence in $3 \mathrm{D}$ systems, and our results confirm his conclusion. No previous work considered the inter relations, among Bloch instability, Stoner instability and dispersion instability. Our work is the only work in the literature connecting all these density-driven electron liquid instabilities within one coherent theoretical framework.

This work is supported by NSF, DARPA, ONR, LPS, and ARO.
1 A. A. Shashkin, S. V. Kravchenko, V. T. Dolgopolov, and T. M. Klapwijk, Phys. Rev. B 66, 073303 (2002).

2 A. A. Shashkin, M. Rahimi, S. Anissimova, S. V. Kravchenko, V. T. Dolgopolov, and T. M. Klapwijk, Phys. Rev. Lett. 91, 046403 (2003).

3 A. A. Shashkin, S. V. Kravchenko, V. T. Dolgopolov, and T. M. Klapwijk, Phys. Rev. Lett. 87, 086801 (2001).

4 O. Prus, Y. Yaish, M. Reznikov, U. Sivan, and V. Pudalov, Phys. Rev. B 67, 205407 (2003).

5 C. L. Chang, S. P. Shukla, W. Pan, V. Venkataraman, J. C. Sturm, and M. Shayegan, Thin Solid Films 321, 51 (1998).

6 E. Tutuc, E. P. De Pootere, S. J. Papadakis, and M. Shayegan, Phys. Rev. Lett. 86, 2858 (2001).

7 E. Tutuc, S. Melinte, and M. Shayegan, Phys. Rev. Lett. 88, 036805 (2002).

8 E. P. De Poortere, E. Tutuc, Y. P. Shkolnikov, K. Vakili, and M. Shayegan, Phys. Rev. B 66, 161308(R) (2002).

9 J. Zhu, H. L. Stormer, L. N. Pfeiffer, K. W. Baldwin, and K. W. West, Phys. Rev. Lett. 90, 056805 (2003).

10 V. M. Pudalov et al., Phys. Rev. Lett. 89, 219702 (2002).

11 V. M. Pudalov, M. E. Gershenson, H. Kojima, G. Brunthaler, A. Prinz, and G. Bauer, Phys. Rev. Lett. 91, 126403 (2003).

12 S. A. Vitkalov, H. Zheng, K. M. Mertes, M. P. Sarachik, and T. M. Klapwijk, Phys. Rev. Lett. 87, 086401 (2001); M. P. Sarachik and S. A. Vitkalov, cond-mat/0209113.

13 V. M. Pudalov, M. E. Gershenson, H. Kojima, N. Butch, E. M. Dizhur, G. Brunthaler, A. Prinz, and G. Bauer, Phys. Rev. Lett. 88, 196404 (2002).

14 Y.-W. Tan, J. Zhu, H. L. Stormer, L. N. Pfeiffer, K. W. Baldwin, and K. W. West, cond-mat/0412260.

15 E. Wigner, Phys. Rev. 46, 1002 (1934).

16 B. Tanatar and D. Ceperley, Phys. Rev. B 39, 5005 (1989).

17 G. Ortiz, M. Harris, and P. Ballone, Phys. Rev. Lett. 82, 5317 (1999).

18 M. F. Bishop and T. McMullen, Phys. Rev. B 62, 15610 (2000).

19 V. V'yurkov and A. Vetrov, Nanotechnology 11, 336 (2000).

20 G. Benenti, G. Caldara, and D. L. Shepelyansky, Phys. Rev. Lett. 86, 5333 (2001).

21 A. Isihara and T. Toyoda, Phys. Rev. B 19, 831 (1979).

22 A. Isihara and Y. Kojima, Phys. Rev. B 11, 710 (1975).

23 D. Y. Kojima and A. Isihara, Phys. Rev. B 20, 489 (1979).

24 I. A. Shelykh, N. T. Bagraev, and L. E. Klyachkin, Semiconductors 37, 1390 (2003).

25 I. A. Shelykh, N. T. Bagraev, and L. E. Klyachkin, Phys. of the Solid State 45, 2189 (2003).

26 A. W. Overhauser, Phys. Rev. 128, 1437 (1962).

27 S. Misawa, Phys. Rev. 140, A1645 (1965).

28 B. S. Shastry, Phys. Rev. B 17, 385 (1978).

29 S. Yarlagadda and G. F. Giuliani, Phys. Rev. B 38, 10966
(1988).

30 S. Yarlagadda and G. F. Giuliani, Phys. Rev. B 40, 5432 (1989).

31 S. Yarlagadda and G. F. Giuliani, Phys. Rev. B 49, 14188 (1994).

32 J. Janak, Phys. Rev. 178, 1416 (1969).

33 S. Doniach and S. Engelsberg, Phys. Rev. Lett. 17, 750 (1966).

34 D. Coffey and K. S. Bedell, Phys. Rev. Lett. 71, 1043 (1993).

35 D. Coffey and C. J. Pethick, Phys. Rev. B 37, 1647 (1988).

36 Y. Zhang and S. Das Sarma, Phys. Rev. B 70, 035104 (2004).

37 Y. Zhang, V. M. Yakoevnko, and S. Das Sarma, condmat/0410039; to appear in Phys. Rev. B .

38 A. A. Abrikosov, L. P. Gor'kov, and I. E. Dzyaloshinski, Methods of quantum field theory in statistical physics (Dover Publications, New York, 1963); G. D. Mahan, Many-Particle Physics (Plenum Press, New York, 1981); A. L. Fetter and J. D. Walecka, Quantum theory of manyparticle systems (McGraw-Hill, San Francisco, 1971).

39 F. Bloch, Z. Phys. 57, 545 (1929).

40 E. C. Stoner, Proc. Roy. Soc. A 165, 372 (1938); A 169, 339 (1938).

41 S. Das Sarma, V. M. Galitski, and Y. Zhang, Phys. Rev. B 69, 125334 (2004); Ying Zhang and S. Das Sarma, Phys. Rev. B 70, 035104 (2004).

42 T. M. Rice, Ann. Phys. (N. Y.) 31, 100 (1965).

43 J. Hubbard, Proc. Roy. Soc. A240, 539 (1957); A243, 336 (1957).

44 L. Hedin, Phys. Rev. 139 (1965).

45 T. Ando, A. B. Fowler, and F. Stern, Rev. Mod. Phys. 54, 437 (1982).

46 Y. Zhang and S. Das Sarma, cond-mat/0408335.

47 F. H. Zong, C. Lin, and D. M. Ceperley, Phys. Rev. E 66, 036703 (2002).

48 C. Attaccalite, S. Moroni, P. Gori-Giorgi, and G. B. Bachelet, Phys. Rev. Lett. 88, 256601 (2002).

49 E. H. Hwang and S. Das Sarma, Phys. Rev. B 64, 165409 (2001).

50 S. Das Sarma, V. M. Galitski, and Y. Zhang, Phys. Rev. B 69, 125334 (2004); Y. Zhang and S. Das Sarma, Phys. Rev. B 70, 035104 (2004); V. M. Galitski and S. Das Sarma, Phys. Rev. B 70, 035111 (2004); A. V. Chubukov and D. L. Maslov, Phys. Rev. B 68, 155113 (2003).

51 J. P. Eisenstein, L. N. Pfeiffer, and K. W. West, Phys. Rev. B 50, 1760 (1994).

52 A. K. Rajagopal and J. C. Kimball, Phys. Rev. B 15, 2819 (1977); A. K. Rajagopal, S. P. Singal, M. Banerjee and J. C. Kimball, Phys. Rev. B 17, 2262 (1978).

53 S. Yarlagadda and G. F. Giuliani, Phys. Rev. B 49, 14172 (1994).

54 G. E. Santoro and G. F. Giuliani, Phys. Rev. B 39, 12818 
(1989). 